Cahiers
de la Recherche
sur les Droits Fondamentaux
Cahiers de la recherche sur les droits fondamentaux

4 | 2005

Quel avenir pour la laïcité cent ans après la loi de 1905 ?

\title{
Laïcités française et américaine en miroir
}

\section{Blandine Chelini-Pont}

\section{(2) OpenEdition}

Journals

Édition électronique

URL : https://journals.openedition.org/crdf/7323

DOI : $10.4000 /$ crdf. 7323

ISSN : 2264-1246

Éditeur

Presses universitaires de Caen

Édition imprimée

Date de publication : 1 décembre 2005

Pagination : 107-118

ISBN : 2-84133-250-0

ISSN : $1634-8842$

Référence électronique

Blandine Chelini-Pont, "Laïcités française et américaine en miroir », Cahiers de la recherche sur les droits fondamentaux [En ligne], 4 | 2005, mis en ligne le 15 décembre 2020, consulté le 14 novembre 2022. URL : http://journals.openedition.org/crdf/7323 ; DOI : https://doi.org/10.4000/crdf.7323 


\title{
Laïcités française et américaine en miroir
}

\author{
Blandine CHELINI-PONT ${ }^{1}$
}

I. Convergences

A. La liberté de conscience comme fondement

B. Non-confessionnalité de l'État et séparation

C. La singularité franco-américaine face aux autres démocraties occidentales

II. Divergences
A. Gallicanisme français
B. La laïcité comme religion civile
C. Confessionnalisme américain

II. Deux laïcités en concurrence?

Un collectif récemment publié pose dans son titre et dans celui d'un de ses articles l'affirmation, originale pour des lecteurs français plutôt sensibles à la forte religiosité de la population et de la classe politique américaines, selon laquelle les États-Unis sont un pays laïque ${ }^{2}$. Protégés par un tel antécédent, lui-même étayé d'un contenu substantiel, nous avons repris à notre tour cette idée pour établir une comparaison entre la France et les États-Unis en matière de définition de l'État et de mode de relations entre ce dernier et les religions que pratiquent ses citoyens. Après une première partie consacrée à récapituler les ressemblances indéniables qui existent entre ces deux pays et qui correspondent à notre vocable de laïcité, nous présenterons leurs différences, facilement discernables sous des principes identiques, et tenterons en conclusion de nous interroger, à la lumière de l'actualité, sur l'avenir de ces deux laïcités, comme corollaire d'une image internationale concurrente entre la France et les États-Unis.

\section{Convergences}

Selon les principes constitutionnels de ces deux démocraties libérales que sont la France et les États-Unis, la perception de leurs valeurs de liberté et de neutralité est parallèle. Ces républiques ont les deux plus anciennes traditions de constitutions écrites. La première constitution française date de 1791, bien avant toutes les autres constitutions écrites européennes. Les autres États ont repris les deux textes les plus anciens sur les droits de l'homme, tous deux écrits en 1789 : la Déclaration française des droits de l'homme et du citoyen et le Bill of Rights américain. Ces textes furent rédigés à quelques semaines d'intervalle. Si les Français tirent fierté de l'antériorité, dans la rédaction et l'application, de leur texte, les Américains se targuent d'avoir appliqué scrupuleusement leur Bill of Rights depuis le 15 décembre 1791, et cela sans interruption. Les principes franco-américains - des droits inhérents de l'homme-

1. Docteur en droit, docteur en histoire contemporaine, maitre de conférences et directrice-adjointe du laboratoire Droit et mutations sociales de la Faculté de droit et de science politique d'Aix-en-Provence, responsable du master Laïcité et droit des cultes. Vient de publier, en collaboration avec J. Gunn, Dieu en France et aux États-Unis : quand les mythes font la loi, Paris, Berg International, 2005.

2. C. Froidevaux-Metterie, «Les États-Unis, une République laïque», in La Conception américaine de la laïcité, E. Zoller (dir.), Paris, Dalloz, 2005, p. 8997. 
ont été depuis inscrits (même s'ils ne sont pas toujours respectés) dans presque toutes les constitutions écrites du monde, ainsi que dans les textes de référence des instances internationales et de nombreuses ONG. Français et Américains tirent donc fierté de leurs déclarations de référence qu'ils considèrent comme exemplaires pour le reste du monde. Le président Bush déclarait le 18 mai 2001 à l'occasion de la reconnaissance de l'indépendance de Cuba: «Les peuples opprimés dans le monde doivent savoir cela des États-Unis. Nous serons toujours le leader mondial pour défendre les droits de l'homme ", tandis que le 17 décembre 2003, dans son discours à propos de la laïcité, le président Chirac déclarait en écho : «Partout dans le monde, la France est connue comme le pays des Droits de l'homme.»

\section{A. La liberté de conscience comme fondement}

Parmi ces droits de l'homme, la liberté de conscience est un fondement que ces deux nations partagent depuis la fin du XVIII ${ }^{\mathrm{e}}$ siècle et qui s'applique précisément à la conviction religieuse. La clause dite de libre-exercice qui termine le premier amendement du Bill of Rights enjoint au Congrès de «ne faire aucune loi ayant pour effet d'interdire le libre-exercice d'une religion ». Elle vise très précisément le droit reconnu de pratiquer la religion de son choix. Elle est équivalente au principe posé par l'article I de la Constitution de 1958 «Elle [la République] respecte toutes les croyances », quand l'Ancien Régime n'en respectait qu'une, et équivalente également au principe de l'article I de la loi du 9 décembre 1905, « La République assure la liberté de conscience, elle garantit le libre exercice des cultes ", tandis que le système précédent, dit concordataire, l'encadrait strictement dans l'espace public.

La philosophie sous-jacente de la liberté de conscience paraît dans les deux cas issue du même libéralisme critique des Lumières ${ }^{3}$, grâce auquel Jefferson comme Voltaire pouvaient défendre que la religion est avant tout une affaire de conscience, une affaire privée, personnelle, qui ne doit être en aucun cas imposée, et qu'on ne peut empêcher chez autrui, quelle que soit sa forme et son contenu. S'il est possible de distinguer les racines protestantes de la liberté de conscience américaine (la foi est un acte volontaire, si elle est contrainte par l'ordre politique elle est inefficace) des racines rationalistes de la liberté de conscience française (la foi ne peut être démontrée, ce qui rend vaine et injuste son imposition), il reste que, quand un Américain pense liberté de conscience, le Français lui répond à l'unisson. Dans les deux cas, la liberté de conscience est un refus, une résistance à subir une vérité imposée de l'extérieur, et le fondement de toute l'organisation sociale. De ce point de vue Roger Williams obtenant par charte royale en 1663 l'absolue liberté de conscience pour la colonie qu'il dirige, préfigure les revendications de Pierre Bayle dans son Commentaire philosophique de $1686^{4}$ et la réflexion de cause à effet que John Locke établit en 1689 entre liberté de conscience et libre-exercice de la religion dans sa fameuse Lettre sur la tolérance 5 .

\section{B. Non-confessionnalité de l'État et séparation}

Il est également aisé de constater combien la France et les États-Unis ont une véritable proximité de conception quant à l'attitude « religieuse » de leur État : ils se ressemblent dans leur absence fondatrice de confessionnalité et de normativité directement confessionnelle, ce qui pour le sociologue et historien Jean Baubérot correspond au premier seuil d'une laïcité réalisée ${ }^{6}$. Ferdinand Buisson, dans son célèbre article «Laïcité» du Dictionnaire de pédagogie, définit «l'État laïque » comme un «État neutre entre les cultes, indépendants de tous les clergés, dégagés de toute conception théologique ». Le dictionnaire Grand Robert la définit, dans son édition de 1992, comme « une notion politique incluant la séparation de la société civile et de la société religieuse, l'État n'exerçant aucun pouvoir religieux et les Églises n'exerçant aucun pouvoir politique ». Le terme « laïcité » évoque donc fondamentalement la non-confessionnalité de l'État et l'indépendance absolue du pouvoir politique dans son fonctionnement et son devenir autonome vis-à-vis de toute forme d'inclusion religieuse. Jules Ferry utilisait d'ailleurs le terme de laïcité dans le champ scolaire comme synonyme de «neutralité confessionnelle ». La laïcisation de l'école publique était en quelque sorte une conséquence inévitable de celle du «pouvoir civil [qui] constitue le régime sous lequel nous vivons depuis 1789 ".

Si le premier seuil de laïcité existe dès lors que la citoyenneté et l'appartenance religieuse sont dissociées, que le droit commun n'a pas de lien de subordination ou de source directe dans l'injonction divine et le droit religieux, que l'union fusionnelle entre l'État et l'Église est rompue et qu'il n'existe plus de religion nationale, d'Église officielle, seuls les États-Unis et la France répondent à cette condition de la laïcité parmi les démocraties occidentales.

Son contenu a été dégagé en France par la voie législative des grandes lois scolaires des années 1880 et par la loi de séparation des Églises et de l'État de 1905, qui en finit avec le système d'une administration directe des cultes en

3. Cf. J. Rawls, qui, dans la lignée de Locke, Rousseau et Kant, fait du principe d'égale liberté de conscience le premier principe de justice nécessaire à l'établissement d'une société, in Théorie de la Justice, trad. fr. C. Audard, Paris, Seuil, 1987, p. 240-257.

4. J.-M. Gros, «Bayle : de la tolérance à la liberté de conscience», in Les Fondements philosophiques de la tolérance, Y.-C. Zarka (dir.), Paris, PUF, 2002, t. I, p. 295-311.

5. Republiée chez Nathan en 2003. Texte qui inspira directement Madison, le Père de la Constitution américaine, dans son idée que la liberté religieuse devait être un droit inaliénable et naturel de chaque citoyen. Sur le développement de la liberté de conscience aux États-Unis, voir J. Van Til, Liberty of Conscience: The History of a Puritan Idea, New Jersey, Nutley, 1972.

6. Voir, entre autres, dans sa très abondante bibliographie, son article sur la laïcité, in Dictionnaire d'éthique et de philosophie morale, $4{ }^{\mathrm{e}}$ éd., Paris, PUF, 2004, p. 1059-1066. 
« concordance » avec l'État ${ }^{7}$. La séparation de la République a été consacrée constitutionnellement à partir du substrat de 1905, aussi bien en 1946 qu'en 1958. De leur côté, les États-Unis sont constitutionnellement un État «laïque », qui pratiquent l'absence conjointe de religion nationale et d'État confessionnel, et séparent l'État de toute forme de religion organisée ${ }^{8}$. Depuis 1787 , la clause de non-établissement du premier amendement du Bill of Rights oblige le Congrès « à ne faire aucune loi établissant une religion quelconque comme religion d'État " ${ }^{9}$. Cette prohibition est similairement posée dans l'article 2 de la loi du 9 décembre 1905: «La République ne reconnaît aucun culte».

Également, à l'article VI (3) de la Constitution fédérale qui prévoit qu' " aucune profession de foi religieuse ne sera exigée comme condition d'aptitude à quelque fonction ou charge publique de confiance dépendant des ÉtatsUnis ", répond l'article 6 de la Déclaration des droits de l'homme et des citoyens, « tous les citoyens sont également admissibles à toutes dignités, places et emplois publics, selon leurs capacités et sans autre distinction que celles de leurs vertus et de leurs talents », ainsi que le principe du préambule de la Constitution de 1946, «nul ne peut être lésé de son emploi en raison de ses origines, de ses opinions et de ses croyances ». On connaît également de Thomas Jefferson la célèbre formule du « mur de séparation ${ }^{10}$ qui vient imager cette réalité constitutionnelle américaine, plus précoce encore que les premières tentatives françaises des années $1790^{11}$.

Quel autre pays - démocratique -, à part la France, partage-t-il avec les États-Unis cette absence fondamentale de confessionnalité de l'État? Quel pays a-t-il dans son histoire utilisé le concept de séparation pour assurer la «liberté souveraine de l'État » contre un cléricalisme domi- nateur, anglican d'un côté, catholique de l'autre? Qui peut, mieux que l'Amérique, comprise comme somme de ses citoyens, comprendre la méfiance française face aux religions inclusives, qui cherchent la symphonie ou l'accommodement préférentiel avec l'État? Quel autre système constitutionnel occidental refuse-t-il aussi fermement l'établissement-reconnaissance de la religion par l'État?

\section{La singularité franco-américaine face aux autres démocraties occidentales}

Où que l'on se tourne sur le continent européen la similitude franco-américaine frappe par sa singularité. L'Europe reste un conservatoire d'époques révolues mais de tenace mémoire. Elle comporte encore des États à prétention confessionnelle comme l'État grec - dont certains des traits se retrouvent dans d'autres pays à majorité orthodoxe, comme la Bulgarie ou Chypre-dont le fondement orthodoxe se marque dès la Constitution, promulguée sous les auspices de la "Très Sainte Trinité ». Cette dernière est présente également dans le serment du président de la République grecque. Le statut de l'Église orthodoxe est celui d'une religion d'État, tempérée par une tolérance juridique très stricte vis-à-vis des minorités musulmanes et catholiques ${ }^{12}$. L'éducation orthodoxe de la jeunesse est obligatoire, les forces armées sont placées sous le patronage de la Vierge Marie et l'Église orthodoxe bénéficie de nombreux soutiens, directement financiers, de la part de la puissance publique. La liberté de conscience est limitée par la répression pénale du prosélytisme ${ }^{13}$, dont le but consiste à fixer en l'état la répartition des appartenances religieuses ${ }^{14}$. Certains craignent que ce modèle se renforce avec l'évolution de la Russie contemporaine où

7. Sur l'administration des cultes au XIX ${ }^{\mathrm{e}}$ siècle, voir J.-M. Léniaud, L'Administration des cultes pendant la période concordataire, Paris, Nouvelles Éditions latines, 1988. Sur la séparation de 1905, voir A. Boyer, 1905. La séparation Églises-État, de la guerre au dialogue, Paris, Cana, 2004, et M. Larkin, L'Église et l'État en France, 1905 : la crise de la séparation, Toulouse, Privat, 2004.

8. Sur cette séparation l'une des synthèses récentes est celle de P. Hamburger, Separation of Church and State, Cambridge, Harvard University Press, 2002, qui a fait l'objet d'un long travail critique de la part d'A. M. Samaha, «Separation of Church and State », Constitutional Commentary, vol. 19, 2002.

9. Sur les motifs historiques du disestablishment, voir la synthèse extrêmement complète d'E. Zoller, «La laïcité aux États-Unis ou la séparation des Églises et de l'État dans la société pluraliste», in La Conception américaine de la lä́cité, p. 3-32, et de M.A. Hamilton, «Les origines religieuses de la clause américaine de non-établissement », p. 71-88 du même ouvrage. Voir également, L. W. Levy, The Establishment Clause: Religion and the First Amendment, Chapel Hill, University of North Carolina Press, 1994; D. O Conkle, Constitutional Law, The Religion Clauses, New York, Foundation Press and Thomson West, 2003.

10. «En énonçant que la législature ne pourrait faire aucune loi établissant aucune religion [...], le peuple américain a voulu rétablir un mur de séparation entre l'Église et l'État », Lettre de Thomas Jefferson du $1^{\text {er }}$ janvier 1802 à l'Association de confession baptiste de Danburry, extrait de ses Écrits, (Writings, New York, The Library of America, 1984, p. 510), analysée par D.L. Dreisbach, « Sowing Useful Truths and Principles : the Danbury Baptists, Thomas Jefferson and the Wall of Separation ", Journal of Church and State, vol. 39, 1997. Dreisbach en a tiré un livre en 2002 au titre identique (New York University Press). D'autres articles au même intitulé: "Thomas Jefferson and the Wall of Separation between Church and State», de D. Davis, Journal of Church and State, vol. 5, 2003, de D. G. Smith, Harvard Journal of Law and Public Policy, vol. 22, 2003, et de M.Y. Hanley, Journal of Southern History, vol. 70, 2004.

11. Sur l'histoire de ces années 1790 et les tentatives de séparation, voir de l'historien et sociologue É. Poulat, Notre laïcité publique, Paris, Berg International, 2003, le chapitre «La catholicité dont nous venons », p. 37-65.

12. Article 3, Section II de la Constitution grecque du 11 juin 1975.

13. Article 13 de cette même Constitution.

14. F. Messner, «Le statut des confessions religieuses dans les pays du sud de l'Europe », in États, religions et liberté religieuse en Méditerranée. Histoire, actualité, prospectives, B. Chelini-Pont (dir.), Aix-en-Provence, Presses universitaires d'Aix-Marseille, 2002, p. 137-147; N. Alizivatos, «Pratiques discriminantes en Europe méditerranéenne : l'exemple grec », in Quelle politique religieuse en Europe et en Méditerranée? Enjeux et perspectives, B. CheliniPont (dir.), Aix-en-Provence, Presses universitaires d'Aix-Marseille, 2004, p. 83-89; T. Fortakis, «Le régime administratif des cultes en Grèce", Annuaire européen d'aministration publique, t. XXII, 2000, p. 139-153; C. Papasthasis, «État et Églises en Grèce», in État et Églises dans l'Union européenne, G. Robbers (dir.), Baden-Baden, Nomos Verlagsgeselleschaft, 1997, p. 79-97. Voir également le contenu du colloque itinérant sur la notion d’Église nationale ( $4^{\mathrm{e}}$ colloque tenu à Thessalonique, 9-10 novembre 2002), J.-P. Durand, B. Basdevant-Gaudemet et C. Papsthasis (dir.), sur L'État et les cultes en Grèce, les identités religieuses dans la République hellénique, Actes parus dans l'Année canonique, $\mathrm{n}^{\circ}$ 45, 2003. 
l'on observe un certain retour du rôle politique de l'Église orthodoxe et des limitations législatives apportées au pluralisme ${ }^{15}$. Cependant la situation est largement variée dans le monde orthodoxe ${ }^{16}$ et les pays à forte majorité orthodoxe, promus à rentrer dans l'Union européenne, comme la Bulgarie et la Roumanie ${ }^{17}$, ou en transition démocratique comme l'Ukraine et la Moldavie, semblent particulièrement influencés par le modèle de reconnaissance des groupes religieux accompagnée d'une absence individuelle de contrainte spirituelle, modèle qui marque les pays d'Europe occidentale ${ }^{18}$.

En effet, même s'ils ont cessé d'être " confessionnels », les États d'Europe occidentale ne pratiquent pas la «séparation » à la façon franco-américaine ${ }^{19}$. La gradation des situations varie de la reconnaissance d'une Église nationale à une Église établie ${ }^{20}$, de la reconnaissance d'Églises et de mouvements précis qui sont toujours considérés comme « réalités parallèles » par l'État ${ }^{21}$ au système de l'enregistrement confessionnel ${ }^{22}$. Le système de reconnaissance / établissement reste très répandu. Le degré de laïcisation se vérifiera alors en fonction du niveau de positivisme du droit commun, fondé sur les respects des libertés individuelles et d'absence d'imbrication de droit religieux dans l'élaboration des normes et des lois.

Ainsi les systèmes européens dits «laïco-céphales » hérités de l'époque moderne. De l'Encyclopédie de Diderot au Grand Dictionnaire universel de Pierre Larousse, on retrouve le terme de «laïcocéphale » à propos de pays où l'Église nationale se trouve dirigée par un laïc. En GrandeBretagne, l'existence d'une Église établie - anglicane n'est pas remise en cause, car elle s'accompagne d'un libre- exercice extrêmement large ${ }^{23}$. Significativement, les systèmes laïcocéphales d'Europe qui maintiennent l'établissement d'une religion ont en même temps gardé un régime monarchique très tempéré, comme au Danemark, où les évêques ont été fonctionnarisés au temps de la Réforme luthérienne. Aujourd'hui l'Église luthérienne reste l'Église nationale et symbolise l'identité du pays alors même que la société danoise est très sécularisée : $87 \%$ des habitants déclarent être affiliés à l'Église luthérienne, mais seuls $12 \%$ vont au temple moins d'une fois par mois. L'État danois soutient directement l'Église luthérienne à hauteur de $11 \%$ de ses revenus quand le reste provient d'un impôt d'Église acquitté par l'ensemble des membres affiliés. D'autres communautés religieuses sont reconnues par l'État danois, protestante, catholique et orthodoxe, judaïsme, Témoins de Jéhovah, bahaïs et sikhs qui représentent $2 \%$ de la population. La liberté de conscience et l'absence de discrimination selon l'origine ou la croyance sont garanties de façon effective par un dispositif très démocratique. Au total, une société fortement sécularisée s'arrange d'un État non laïcisé ${ }^{24}$.

Certes, la sécularisation sans séparation doit faire face à un pluralisme religieux plus accentué, une indifférence et un agnosticisme plus marqués. L'évolution des autres pays scandinaves semble prouver que les systèmes du nord de l'Europe se dirigent vers la séparation. L'Église luthérienne de Suède n'est plus une Église nationale depuis l'année 2000 et un changement de même nature s'opère en Norvège ${ }^{25}$. La situation finlandaise évolue également puisque le président de la République ne nomme plus les évêques ${ }^{26}$.

15. J. Gunn, "The Law of the Russian Federation on the Freedom of conscience and Religious Associations», H. Berman, «Freedom of Religion in Russia », L.A. Uzzell, «Federal and Provincial Religious Freedom Laws in Russia », in Proselytism and Orthodoxy in Russia. The New War for Souls, J. Witte Jr et M. Bourdeaux (dir.), Maryknoll, Orbis books, 1999, p. 239 à 323. Cf. également M. Bourdeaux, "The Former Soviet Union: Trends in Religious Liberty", in Religious Freedom in the World: A Global Report on Freedom and Persecution, 35, 42, P. Marshall (éd.), Nashville, Broadman \& Holman Publishers, 2000; A. Lekhel, «Leveling the Playing Field for Religious "Liberty” in Russia : A Critical Analysis of the 1997 Law "On Freedom of Conscience and Religious Associations" ", Vanderbilt Journal of Transnational Law, vol. 32, 1999.

16. L. Beauvisage, La Croix et la Faucille, la religion à l'épreuve du post-soviétisme, Paris, Bayard, 1999; F. Thual, Le Douaire de Byzance. Territoires et identités de l'orthodoxie, Paris, Ellipses, 1998.

17. Colloque itinérant sur la notion d'Église nationale ( $3^{\mathrm{e}}$ colloque tenu à Cluj, 10-11 mai 2002), sur Le Régime des cultes en Roumanie, Actes parus dans l'Année canonique, $\mathrm{n}^{\circ}$ 45, 2003.

18. Law and Religion in Post-Communist Europe, S. Ferrari et W. Cole Durham (dir.), Louvain, Peeters, 2003; Laws on Religion and the State in Post-Communist Europe, W. Cole Durham et S. Ferrari (dir.), Louvain, Peeters, 2004; Religion and patterns of social transformation, D. Marinovic Jerolinov (dir.), Zagreb, Institute for Social Research, 2004.

19. Church and State in Contemporary Europe: The Chimera of Neutrality, J.T.S. Madeley et Z. Enyedi (éd.), Londres - Portland, Frank Cass, 2003.

20. Colloque itinérant sur la notion d'Église nationale ( $1^{\mathrm{er}}$ colloque tenu à l'Institut catholique de Paris, 6-7 décembre 200o) sur La Notion «d'Église nationale» en Europe, Actes parus dans l'Année canonique, nº 43, 2001, p. 7-118.

21. J. Baubérot, "Modèles de société et politique des cultes en Europe», in Quelle politique religieuse en Europe et en Méditerranée?..., p. 83-89; W. Cole Durham, "Perspectives on Religious Liberty: A Comparative Framework », in Religious Human Rights in Global Perspective : Legal Perspectives, J.D. van der Vyver et J. Witte Jr (dir.), La Haye - Boston, Martin Nijhoff Publishers, 1996.

22. Système défendu, à titre d'exemple, par l'État hongrois actuel: «The philosophy of our church policy is based on three cardinal tenets. The first regards the recognition that the church is sui generis reality. Therefore the church is not a sub-unit of the apparatus of state institutions but a reality, which is equal with the state [...]. The second assumption is that the separation of church and State does not mean the separation of church and citizens of the Hungarian nation. The proper attitude is not confrontation but a harmonic cooperation. Our third fundamental point is that we wish to recognize the church's service in its totality" (Préface du livret Legislation on Church - State Relations in Hungary, B. Schanda (éd.), Budapest, Ministry of Cultural Heritage, 2002, p. 7, cf. également l'article sur les relations État-Églises en Autriche, de R. Potz, dans le collectif dirigé par G. Robbers, État et Églises dans l'Union européenne, p. 247-279).

23. D. MacClean, «État et Églises au Royaume-Uni », in État et Églises dans l’Union européenne, p. 331-348; P.A. Diaper, Law and Religion in England between 1532-1994: The Legal Development of the Established Church, Religious Toleration and Conscientious Objection, Th. Droit canonique, Rome, Pontificia Università della Santa Croce, Facultà di diritto canonico, 2000 ; colloque itinérant sur la notion d'Église nationale $\left(2^{\mathrm{e}}\right.$ colloque tenu à Cardiff, $13-14$ septembre 2001), N. Doe (dir.), sur L'Église anglicane en Grande-Bretagne, Actes parus dans l'Année canonique, $\mathrm{n}^{\circ}$ 44, 2002.

24. I. Dübeck, «État et Églises au Danemark », in États et Églises dans l'Union européenne, p. 39-59.

25. R. Schött, «État et Églises en Suède», ibid., p. 317-329.

26. Depuis le début des années 200o, voir M. Heikkiklä et al., «État et Églises en Finlande », ibid., p. 301-315. 
Tous les pays qui ont ou qui ont eu jusqu'à une période très récente une Église nationale ou une Église établie sont en même temps des monarchies constitutionnelles. En quittant ce régime ou en le conservant, certains ont pu évoluer vers un système de reconnaissance pluraliste entre les religions et l'État et perdre leur lien officiel avec une religion mais pas leur système de contrat hérité de leur ancienne confessionnalité catholique. La Belgique est un exemple significatif. L'État belge se veut neutre à l'égard des religions et affirme n'être soumis à aucune tutelle ecclésiastique. Inversement, il s'interdit toute ingérence dans les questions doctrinales et les affaires intérieures des différents cultes ${ }^{27}$. Certains Belges parlent en conséquence de séparation entre l'État et les cultes et de l'État laïque. Mais la Belgique pratique un système pluraliste de six cultes reconnus, les cultes catholique, protestant, anglican, orthodoxe, israélite et musulman. Acte officiel de l'État, la « reconnaissance» d'un culte induit une protection particulière accordée en raison d'une «utilité sociale». Cela signifie que le culte en question est jugé répondre au besoin social d'une fraction de la population. En découlent certains avantages comme la prise en charge des traitements des ministres du culte, la présence d'aumôniers rémunérés par les pouvoirs publics dans les hôpitaux, les prisons, les aéroports et l'armée, et, pour la plupart des cultes reconnus, l'existence - chaque fois que le nombre d'élèves atteint un certain seuil - de cours de religion à l'école publique $^{28}$.

De plus, malgré cet interventionnisme pluraliste assez large et une indéniable évolution ces dernières décennies, le poids du catholicisme reste prédominant en Belgique. Il regroupe $55 \%$ de la population et reçoit l'essentiel des fonds publics. On retrouve ce double facteur - pluralisme récent et influence catholique forte - plus nettement encore dans d'autres pays européens catholiques comme l'Italie $^{29}$ et l'Espagne ${ }^{30}$. Les termes de la Constitution espagnole actuelle induisent deux constats ; d'une part la Constitution affirme: «aucune religion ne sera religion d'État»; d'autre part, l'article 16 (3) de la Constitution du 27 décembre 1978 prévoit que «les pouvoirs publics tiendront compte des croyances religieuses de la société espagnole et entretiendront de ce fait des relations de coopération avec l'Église catholique et les autres confessions ». Si le concordat avec l'Église catholique a été modifié, il n'a pas été aboli pour autant. Les accords signés avec le Vatican prévoient une subvention de l'État à l'Église catholique espagnole et à l'enseignement privé catholique qui reste fort, surtout dans le primaire. À ceci s'ajoute une catéchèse facultative dans les établissements publics. 0,5 \% des impôts directs sont - au choix des contribuables - affectés soit au « maintien de l'Église » catholique, soit «à d'autres activités d'intérêt social ». Les fonds recueillis dans le cadre de cette seconde option se trouvent reversés à diverses organisations de la société civile, y compris des associations catholiques.

L'Espagne a évolué à partir du régime d'État confessionnel, celui du national-catholicisme, installé par le général Franco à la fin des années 1930, dans lequel le concordat de 1953 avait induit une situation de complète confessionnalité. Non seulement l'État était confessionnel, mais la religion catholique était considérée comme «l'unique vraie religion», l'enseignement public devait suivre le dogme et la morale catholique, les prélats étaient présents dans les institutions politiques et administratives de l'État, le clergé possédait un statut nettement privilégié et les associations et publications catholiques jouissaient d'un régime spécial, le mariage canonique était civilement obligatoire pour tout catholique et aucune cérémonie ou manifestation religieuse extérieure autre que celles de la religion catholique n'était permise. L'Espagne est passée dans les années 1970 à une déconfessionnalisation de l'État, qui est allée de pair avec la libéralisation et la modernisation de la société espagnole par un droit $^{31}$ commun déconfessionnalisé. Le concordat a été renégocié par les accords de 1979 et d'autres accords de coopération entre l'État et les cultes très minoritaires comme les protestants, les israélites et les musulmans ont été signés en 1992, établissant un pluralisme dans la reconnaissance ${ }^{32}$. Cette situation récente est comparable à l'évolution italienne et portugaise, quoique dans ces deux derniers cas, la confessionnalité catholique de l'État ait été abandonnée depuis plus longtemps ${ }^{33}$.

27. Cour de cassation de Belgique, 20 octobre 1994, Revue critique de jurisprudence belge, 1996, p. 119-129; Cour de cassation de Belgique, Chambres réunies, 3 juin 1999, ${ }^{\circ}$ JC99631.

28. R. Torfs, «État et Églises en Belgique», in États et Églises dans l’Union européenne, p. 15-37; Id., "Autonomy of Churches in Belgium. Status Quaestionis and Current Debate», in Legal Position of Churches and Church Autonomy, H. Warnink (dir.), Louvain, Peeters, 2001, p. 83-110; Id., « Kirche und Staat. Belgien ", in Lexikon für Kirchen- und Staatskirchenrecht, 2, A. von Campenhausen, I. Riedel-Spangenberger, R. Sebott (dir.), Paderborn Munich - Vienne - Zürich, Schöning, 2002, p. 414-419; L.-L. Christians, "Questions nouvelles du régime administratif des cultes en Belgique ", Annuaire européen d'administration publique, t. XXII, 2000, p. 29-76.

29. Pour des références plus complètes sur l’Italie, voir E. Tawil, «Les relations conventionnelles entre l'État et les confessions religieuses en Italie », dans ce même volume.

30. J. Mantecon, «Relation État-religions et liberté religieuse en Espagne », in Quelle politique religieuse en Europe et en Méditerranée?..., p. 73-79.

31. Le débat sur l'opportunité de l'immixtion de l'Église catholique durant l'année 2005 dans les projets de loi du nouveau gouvernement Zapatero montre cependant que la « séparation » à l'espagnole reste très relative pour l'Église catholique. Cf. l'article de J.I. Calleja, «Zapatero y la Iglesia », El Correo, 14 novembre de 2004. Voir également la revue de presse du Courrier international du 25 janvier 2005 sur la question et la première Lettre à l'épiscopat espagnol de Benoît XVI.

32. Confesiones minoritas en Espagna, Guia de entidas y Vademecum normativo, J. Mantecon (dir.), Madrid, Ministerio de Justicia - Direccion general de Asuntos Religiosos, 2003.

33. L'article 8 (3) de la Constitution italienne du 27 décembre 1947 prévoit que les relations des confessions religieuses avec l'État sont « réglées par la loi sur la base d'ententes avec les représentants de chaque confession ». Voir B. Basdevant-Gaudemet, «Organisation cultuelle du XIx siècle à la décolonisation, la façade européenne, des modèles concordataires aux sécularisations aménagées », in États, religions et liberté religieuse en Méditerranée..., p. 15-53, et 


\section{Divergences}

Le paradoxe que nous fait pourtant atteindre ce panorama résulte de ce que malgré d'évidentes ressemblances constitutionnelles sur la neutralité de l'État comprise comme aconfessionnalité de son administration publique et de son corps légal, et malgré l'absence de relations « concordataires » avec les religions au regard de la situation d'autres démocraties occidentales, les États-Unis et la France restent néanmoins très différents dans la gestion légale et jurisprudentielle du fait religieux, en ses dimensions collectives.

Assurément dans les deux cas, la religion comme entité collective s'organise de manière associative et libérale parfaitement indépendante de l'État. Par, la loi commune, l'État américain fournit une structure juridique grâce à laquelle les cultes peuvent s'organiser. La plupart d'entre eux constituent des sociétés à but non lucratif, et certains disposent de trustees pour gérer leur propriété, d'autres étant constitués en associations de droit commun. N'importe quel culte peut s'organiser selon la forme juridique offerte par l'une de ces structures, aucune autorisation préalable n'est requise. L'État n'a pas à statuer sur l'existence d'un culte. Textuellement la France a un système analogique. La religion s'y organise librement, de manière associative, dans le cadre de l'association cultuelle, de la congrégation ou de l'association de droit commun ${ }^{34}$. Dans les deux pays, les citoyens s'organisent librement pour assurer leur entretien spirituel, sans que l'État n'ait la possibilité de les en empêcher, pour autant que cette organisation reste conforme au cadre de la légalité associative fixée par la loi générale ${ }^{35}$.

Et pourtant c'est en grande partie sur la « relation » que l'État entretient avec les associations religieuses et leurs membres que la différence se cristallise. Chaque pays semble partiellement et inversement séparé, le politique organisant et / ou surveillant le religieux dans le cas français, le religieux harcelant le politique dans le cas américain, cependant que les deux États reconnaissent de facto les religions comme partenaires essentiels quand rien ne le laisse prévoir dans leur Constitution.

\section{A. Gallicanisme français}

Le régime de laïcité française conserve un fort habitus gallican (le catholicisme est la religion de l'État qui en est le gardien) et garde de nombreuses traces du système de reconnaissance concordataire d'antan. Quoique farouchement niée, cette réalité rapproche la France de ses voisins européens, qui ont cherché à aménager le même héritage juridico-religieux dans la sécularisation et le pluralisme. De ce point de vue et en comparaison, les États-Unis semblent respecter plus scrupuleusement le principe de séparation que la France ${ }^{36}$. Là où cette dernière admet que la séparation de 1905 ne concerne pas l'ensemble de son territoire $^{37}$, les États-Unis, qu'il s'agisse de l'État fédéral ou des États fédérés, sont parfaitement et uniformément «séparés». Séparé des Églises, l’État américain ne s'intéresse pas aux religions et elles ne peuvent officiellement rien attendre de lui. Cette séparation américaine fait interdiction absolue à l'État d'aider les religions ${ }^{38}$, dans une neutralité encore plus «négative » que la neutralité française en matière scolaire ${ }^{39}$. Les écoles confessionnelles françaises sont soutenues et subventionnées dès lors qu'elles sont sous contrat et l'ensemble du patrimoine ecclésial catholique d'avant 1905 est propriété publique, entretenu par les collectivités publiques et laissé à la disposition du culte catholique. L'enseignement religieux lui-même est davantage protégé dans les écoles publiques françaises qu'aux États-Unis. Il est légal et obligatoirement organisé dans les départements d'Alsace-Moselle dans lesquels ne s'applique pas la loi de 1905. Quand la Cour suprême, par

dans le collectif dirigé par G. Robbers, État et Églises dans l'Union européenne, les contributions de S. Ferrari, «État et Églises en Italie», p. 183-206, et de V. Canas, «État et Églises au Portugal », p. 279-298.

34. «Les institutions cultuelles », Partie V du Traité de droit français des religions, F. Messner, P.-H. Prélot et J.-M. Woerling (dir.), Paris, Litec, 2003, p. 759-862. Par ailleurs, la loi du 2 janvier 2007 rappelle que l'exercice collectif d'un culte peut se passer du régime associatif par le seul fait de la liberté de réunion.

35. Les Français ont tendance à minorer les fortes contraintes légales qui existent aux États-Unis sur les critères d'ordre public ou de droit commun (aussi bien qu'en France). Sur ces limites, voir les travaux de l'European-American Law and Religion Consortium (IVth Symposium, Budapest-Manresa, 4 décembre 2003) sur le thème The Permissible Scope of Legal limitations on Freedom of Religion or Belief: a comparative perspective, en cours de publication dans la Emory Law Review (contributions pour la France: Alain Garay, Blandine Chelini-Pont, Emmanuel Tawil; pour les États-Unis: Frederic Mark Gedicks).

36. Sur la notion de séparation, cf. E. Tawil, «La République ne reconnaît, ne salarie, ne subventionne aucun culte », Annuaire Droit et Religions 2005, à paraître.

37. Il existe en France six régimes des cultes (E. Tawil, note sous CAA Paris, 31 décembre 2003, JCPA, nº 1404, 2004), la plupart ne prévoyant pas de disposition comparable à l'article 2 alinéa $1^{\text {er }}$ de la loi de 1905, selon lequel «la République ne reconnaît, ne salarie, ni ne subventionne aucun culte ». Le Conseil d'État a consacré notamment les régimes de cultes reconnus d'Alsace-Moselle (CE 6 avril 2001, Syndicat national des enseignants du second degré, Revue de droit canonique, 2002, p. 377-416, conclusions E. Mignon, note E. Tawil), de Guyane (CE 9 octobre 1981, Beherec, Rec., p. 358). Le Conseil d'État a également affirmé que, dans certaines circonstances, le principe de laïcité n'interdisait pas un financement public au culte (CE 16 mars 2005 , Ministère de l'outre-mer / gouvernement de Polynésie, req. $\mathrm{n}^{\circ}$ 265560).

38. Ce qui en France n'est pas vrai, puisque par le biais de la fiscalité des cultes (associations cultuelles et congrégations) d'une part, et les possibilités de subventions aux associations d'intérêt général ou d'utilité publique d'autre part, l'État français continue d'assurer une " reconnaissance indirecte » des religions « utiles» sur son territoire. Sur ces questions, cf. A. Garay, «Les régimes fiscaux et leur influence sur les politiques religieuses », in Quelle politique religieuse en Europe et en Méditerranée?..., p. 45-67.

39. La neutralité financière française envers les écoles confessionnelles a été fortement tempérée par la loi Debré sur les contrats d'association avec l'État du 31 décembre 1959 et protégée dans cette tempérance par la jurisprudence du Conseil d'État. Voir notamment la décision d'Assemblée du 6 avril 1990 qui a estimé que les aides à l'enseignement général relevaient toujours de la loi Falloux du 15 mars 1850 aux termes de laquelle « les établissements libres peuvent obtenir des communes, des départements ou de l'État, un local ou une subvention, sans que cette subvention puisse excéder le dixième des dépenses annuelles de l'établissement » (CE, Ass. 6 avril 1990, Département d'Ille-et-Vilaine, rec., p. 91). 
l'arrêt Illinois ex rel. MacCollum v. Board of Education, 333 US 203 (1948), a décidé que l'enseignement religieux serait strictement interdit dans l'enceinte même de l'école publique, le Conseil d'État français a considéré l'enseignement religieux (interprétant le décret de 1881 établissant le droit à l'instruction religieuse dans les collèges et lycées et extirpant ce droit également de la loi de 1905 comme composante de la liberté des cultes) comme un service nécessaire, quand il n'y avait pas d'autre possibilité de l'organiser facilement en dehors de l'école ${ }^{40}$.

Il est également possible de parler d'habitus gallican quand la liberté d'association à la française semble entourée de limitations légales, fiscales et réglementaires qui trahissent une approche laïco-céphale, discriminant fortement tout ce qui n'est pas religion traditionnelle et favorisant de facto la religion catholique ${ }^{41}$ et les religions anciennement instituées, par un système d'exemption fiscale (cf. l'article 795-10 du Code général des impôts), de déclaration de dons et legs et un système de subventions directes ou indirectes aux associations d'origine confessionnelle ayant des activités socialement utiles.

De ce fait, le recours à «l'arme fiscale » est un moyen important pour contrôler par leurs finances des groupes religieux non traditionnels, suspectés de malversations lucratives et de manipulation psychologique sur le dos de leurs adeptes ${ }^{42}$. Par l'acceptation ou le refus du statut strictement cultuel de l'association, par l'acceptation ou le refus de l'intérêt général de l'association de droit commun qui entraîne la possibilité de taxer ou non les revenus de ces associations comme des bénéfices, par les effets fiscaux de l'absence de sectorisation des activités cultuelles et des activités culturelles, le droit français contrôle très strictement l'organisation matérielle des religions en France. Les «sectes » et leur montage associatif sont ainsi devenues, selon le titre du livre de l'ancien rapporteur sur les sectes en France, une «affaire d'État » ${ }^{43}$.

La politique traditionnelle de surveillance-collaboration religieuse de l'État français a donné lieu à une politique publique de luttes contre les mouvements sectaires dans les années 1990 qui s'est soldée par la création d'une mission interministérielle et par une loi contre leurs dérives abusives et frauduleuses, dite «About-Picard» du 12 juin $2001^{44}$.
La séparation de l'État français est enfin relative quand ses pouvoirs publics font du catholicisme, du judaïsme et du protestantisme des interlocuteurs privilégiés du dialogue politique et social ${ }^{45}$, et cherchent également à institutionnaliser au même niveau l'Islam de France, par le biais de rencontres et d'incitations du ministère de l'Intérieur, à organiser un Conseil français du culte musulman. Cette volonté explique la grande tolérance des pouvoirs publics envers la situation légale des associations musulmanes, qui laisse souvent à désirer.

\section{B. La laïcité comme religion civile}

Que l'État français entretienne des relations administratives privilégiées avec certaines religions par l'aide financière, l'incitation fiscale, la formalisation des rencontres entre responsables à tous les échelons administratifs, n'a en soi rien de singulier à l'instar du reste de l'Europe ${ }^{46}$. Nous posons simplement la contradiction qu'il y a à insister si fortement dans le discours commun sur le principe de séparation comme définition de la laïcité, face à une réalité qu'on peut sans difficulté définir comme une politique administrative de partenariat ou de surveillance des cultes, transformant cette séparation théorique en fiction d'ignorance légale, selon l'expression du Doyen Maurice Hauriou.

Dans cette contradiction se renferme en fait un secret de polichinelle. La laïcité est avant tout une «religion civile ", celle que l'État républicain a construite à la fois comme ciment de sa légitimité et de l'identité nationale. Quoique la laïcité se définisse constitutionnellement par les principes de neutralité, de séparation et de liberté de conscience, la somme de ces principes ne rend pas compte de la formidable puissance symbolique qui sous-tend la laïcité comme identité citoyenne. Et dans cette identité, l'État est définitivement débarrassé de la religion dans son principe, son autorité et sa légalité ; le divorce est consommé. Que l'État continue donc à s'intéresser de près à l'évolution de ce phénomène, soit avec bienveillance soit avec méfiance est un non-dit de l'histoire de la laïcité. La guerre scolaire est plus étudiée que les accommodements successifs qui permettent aujourd'hui à un quart des élèves

40. CE Ass, 6 juin 1947, Union catholique des hommes du diocèse de Versailles, rec. p. 250. Ass, $1^{\text {er }}$ avril 1949, Chaneveau et autres, rec. p. 161 ; Sect., 28 janvier 1955, Association professionnelle des aumôniers de l'enseignement public, rec. p. 51. cf. Traité de droit français des religions, VIII ${ }^{\mathrm{e}}$ partie, Enseignement et Religion, p. 1119-1289.

41. P.-H. Prélot, «Les religions et l'égalité en droit français », Revue de droit public, 2001, p. 737-775; D. Latournerie, "Sectes et laïcité », Revue de droit public, 2004, p. 1327-1338.

42. Les Sectes et l'argent, rapport $\mathrm{n}^{\circ} 1687$, commission d'enquête, Assemblée nationale (président Jacques Guyard, rapporteur Jean-Pierre Brard). Le Code général des impôts a été modifié en 1992 en matière de taxation des seuls dons manuels révélés à l’administration. Voir A. Garay et D. Guizard, Les Dons manuels aux associations. Régime juridique et fiscal, Lyon, Éditions Juriservice, 2003.

43. A. Gest, Rapport parlementaire sur les sectes en France, $\mathrm{n}^{\circ}$ 1687, Paris, Éditions Patrick Banon, 1998 ; Id., Les Sectes, une affaire d’État, Paris, Éditions de l'Archer, 1999.

44. Loi $\mathrm{n}^{\circ}$ 2001-504 du 12 juin 2001, JO éd. LD, 13 juin 2001, p. 9337.

45. Par le biais du cadre administratif du régime social des cultes (Commission consultative des cultes auprès du ministre des Affaires sociales) et d'une fiscalité protectrice (associations caritatives reconnues d'utilité publique ou d'intérêt général, susceptibles de dons et legs, de subventions et d'exonérations). Cf. A. Garay, «Religion et fiscalité », in Colloque de l'Académie des sciences morales et politiques, 25-27 avril 2005, www.1905-2005.fr.

46. Un exemple récent de cette évidence se trouve dans le contenu d'une lettre inédite du ministre de l'Intérieur aux préfets de la République du 30 septembre 2004 sur le centenaire de la loi de 1905: «Le Centenaire doit être l'occasion de manifester la qualité des relations régulières que vous entretenez avec les représentants des principaux cultes en abordant avec eux les problèmes qu'ils souhaitent évoquer en matière de mise en œuvre des règles de laïcité dans l'espace public» (référence NOR / INT / A / 04 / o0120 / C). 
français d'être scolarisés dans des établissements confessionnels grâce aux deniers publics...

Qui plus est, le modèle citoyen de la laïcité française est plus inclusif encore que le schéma théorique d'une neutralité totale de l'État. Dans ce modèle, la société ellemême et ses citoyens sont affranchis des interdits que font peser les religions sur les consciences et à l'État neutre correspond le citoyen libre de penser par lui-même. Afin que la liberté de conscience de l'individu et la neutralité de l'État ne soient pas menacées, le degré de visibilité publique et de poids individuel de la religion doit être réduit au minimum autorisé par la loi par «sécurité». L'affaire du voile à l'école, commencée en mai 2003 et soldée par une loi d'interdiction en mars $2004{ }^{47}$, est à cet égard particulièrement illustrative. L'éventuelle visibilité de l'Islam dans l'école républicaine, encadrée depuis 1989 par les avis et la quarantaine d'arrêts du Conseil d'État, a vu se dresser contre elle cette certitude qu'un signe religieux à ce point chargé de sens allait à l'encontre de toute la pédagogie républicaine et de son sens de la liberté et de l'égalité de tous devant la loi.

\section{Confessionnalisme américain}

Face à ce panorama, la laïcité américaine semblerait beaucoup plus respectueuse de l'indépendance du spirituel comme le supposent le principe de neutralité et celui de séparation. C'est d'ailleurs l'opinion que dégage Elizabeth Zoller dans la conclusion de son article précité et qui lui permet d'expliquer l'extraordinaire religiosité du peuple américain : "si la religion imprègne si profondément la société américaine, c’est justement parce que l'État est complètement séparé de la religion, parce qu'il ne s'intéresse pas à elle et que celle-ci se voit privée par la Cour suprême de la possibilité de s'intéresser à lui. En d'autres termes, c'est bien la séparation entre la religion et l'État qui crée et alimente tous les jours la religiosité de la société américaine, comme Tocqueville l'avait noté dans la Démocratie. La France, quoique laïque, est parvenue à des résultats complètement différents de ceux atteints par les États-Unis. La société française n'est nullement pétrie de religiosité comme la société américaine. Comment expliquer cela ? À la lumière de la conception américaine du principe de séparation des Églises et de l'État, on serait tenté de dire que c'est parce que l'État et la religion sont en France beaucoup moins séparés qu'on ne veut bien le dire. Ce n'est pas tant qu'en France, la République aide les Églises bien plus qu'elles ne le sont aux
États-Unis ; c'est bien plutôt qu'en France, la République n'a pu s'affirmer comme "l'association politique" (article 2 de la DDHC) de la France moderne qu'en construisant avec la laïcité, notamment à l'école, une religion civile dont les États-Unis n'ont jamais eu besoin. Les États-Unis n'ont pas de laïcité au second degré; ils n'ont pas de religion civile et à vrai dire, une telle notion est aux antipodes de leur culture. L'État est neutre aux États-Unis, il n'a pas de religion, ou, s'il en a une, c'est celle de ne pas en avoir et de toujours laisser aux individus le droit de se former la sienne ${ }^{48}$.

Cette conclusion nous paraît juste sur la partie française. Mais sur la partie américaine, si l'on admet avec la Professeure Zoller que l'État américain n'a aucune religion civile, il faut néanmoins ajouter qu'il subit de plus en plus la vivace religion civile de sa société. De sorte qu'un danger inverse menace la laïcité américaine par rapport à la laïcité française. Quand l'État semble imposer en France la neutralité religieuse sur sa société et encadrer très fermement l'expression spirituelle de ses citoyens, la société civile américaine semble vouloir réduire l'espace de neutralité de son État et exiger toujours plus de place et de reconnaissance pour le religieux. Si l'État s'interdit aux États-Unis de s'intéresser aux religions, les religions s'intéressent constamment à l'État et aux possibilités que leur offre la loi d'exiger davantage en matière de libertés, de non-discrimination et d'égalité de traitement.

Ainsi, les lois et la jurisprudence récentes semblent tirer l'État américain vers une complaisance toujours plus forte au profit de groupements religieux investissant par le lobbying et les alliances tactiques toutes les allées du pouvoir politique et judiciaire. Certes, la religiosité profonde qui anime les citoyens américains ne signifie pas que l'État américain soit religieux ou le soit devenu. Mais la religion civile américaine, objet de débats et de recherches passionnées depuis l'article écrit par Robert N. Bellah dans la revue Daedalus en $1967^{49}$, quoique de plus en plus spécifi$q^{2} e^{50}$, n'a jamais fait que renforcer l'assurance des groupes religieux dans l'idée que l'État doit favoriser l'expression religieuse comme le ciment spirituel de l'esprit national et de la démocratie. Et si la séparation interdit à l’État de se mêler de la dimension spirituelle de ses citoyens, la société civile l'oblige à faire en sorte qu'elle soit toujours prise en compte et rendue «compatible» avec les exigences de l'organisation commune, par une logique dite d'accommodation $^{51}$. Dans la relation société civile-État, l'exception américaine signifie que l'État appartient à la société civile et ne la dirige pas. Il est son cadre commun ${ }^{52}$. Le moteur

47. Sur un récapitulatif chronologique de l'affaire et un survol de ses différents acteurs, voir B. Chelini-Pont et J. Gunn, Dieu en France et aux États-Unis : quand les mythes font la loi, chapitre $\mathrm{V}$.

48. E. Zoller, «La laïcité aux États-Unis ou la séparation des Églises et de l'État dans la société pluraliste », p. 32.

49. Robert N. Bellah est, avec Sidney E. Mead, le principal « concepteur » de l'idée de religion civile américaine. La première occurrence de l'expression fut son article "Civil Religion in America ", Daedalus, 96, hiver 1967, p. 1-21; puis "American Civil Religion in the 1970's", Anglican Theological Review, Supplementary Series, I, juillet 1973, p. 8-20 ; «Reflections on Reality in America », Radical Religion, I, été-automne 1974, p. 38-49; « Religion and Polity in America ", Andower Newton Quarterly, 15, novembre 1974; et enfin son ouvrage The Broken Covenant: American Civil Religion in Time of Trial, New York, Seabury Press, 1975 .

50. Cf. S. Fath, Dieu bénisse l'Amérique, la religion de la Maison Blanche, Paris, Seuil, 2004.

51. L. Mayali, Le Façonnage juridique du marché des religions aux États-Unis, Paris, Mille et une nuits, 2002.

52. É. Duquesnoy, «Et pourtant elle tient - la mosaïque américaine», in L’Exception américaine, P. Gauchon (dir.), Paris, PUF, 2004, p. 31-43. 
relationnel entre les deux entités entraîne la société civile à faire progresser ses marges de liberté aux dépends de la marge non confessionnelle de son État.

Certes, un profond effort de neutralité a été fourni après l'arrêt de principe Everson de $1947^{53}$, pour considérer à égalité toutes les religions ${ }^{54}$ et réduire l'influence dominante du christianisme, en déclarant inconstitutionnelle la prière confessionnelle et le catéchisme à l'école publique $^{55}$, mais également le contenu créationniste de l'enseignement de la biologie et de l'histoire ${ }^{56}$ dans ces mêmes écoles publiques, jusqu'au contenu trop religieux des décorations publiques à Noël ${ }^{57}$ ! L'histoire est également fournie qui a porté la Cour suprême à interdire les comportements religieux dérogatoires à la loi commune ${ }^{58}$. Mais il n'en reste pas moins que l'on peut être surpris, depuis le côté français du miroir, par la grande souplesse de la tradition de balancing text ou de reasonable accomodation utilisée par la Cour suprême (qui correspondrait au principe de proportionnalité) en cas de tension entre règles religieuses, droit du travail, droit scolaire et autres ${ }^{59}$, ainsi que par le réflexe fréquent des groupes religieux et militants, en cas de jurisprudence trop « neutre », de faire appel aux parlementaires pour qu'ils adoptent une loi qui réassure la liberté religieuse du citoyen. Ainsi, après l'arrêt Smith de $1990^{60}$, confirmant le renvoi de deux Amérindiens employés par l'État d'Oregon qui fumaient du peyotl « liturgique» pendant leur temps de travail, la réponse des représentants de la Nation fut le vote du Religious Freedom Restauration Act en 1993, minorant à ce point la question du compelling interest, que la Cour dut réintervenir en 1997, à l'occasion d'une nouvelle jurisprudence, pour déclarer cette loi inconstitutionnelle ${ }^{61}$ !

En ce qui concerne l'enseignement libre, la jurisprudence de la Cour suprême est également passée d'une doctrine "séparationiste » très stricte dans les années $1940^{62}$ - récapitulée dans l'arrêt de principe Lemon v. Kurtzman de $1971^{63}$ qui confirma l'interdiction de toute forme de financement, même à des fins séculières, d'institutions publiques vis-à-vis des écoles libres ${ }^{64}$ - à une doctrine apparemment plus souple à partir des années 1980, sous l'effet d'une interprétation moins « crispée » du principe de neutralité financière de l'État, sur le critère de l'égalité et de la non-discrimination entre citoyens, dans l'octroi d'aides nécessaires à tous les enfants quand il s'agit de matériel pédagogique ${ }^{65}$, de bourses quand il s'agit d'inscription scolaire ${ }^{66}$, de personnel payé par l'État quand il s'agit d'enseignement très spécialisé ${ }^{67}$. Même si nous sommes encore loin du double système scolaire géré par l'État français depuis 1959, sous l'effet conjugué de la lutte des années 1960 pour les droits civils contre la discrimination, les juridictions américaines sont devenues beaucoup plus sensibles au principe d'égalité entre les enfants et cherchent les voies d'une " accommodation » vis-à-vis du choix éducatif des parents ${ }^{68}$ qui favorisent un respect

53. « Ni l'État ni le gouvernement fédéral ne peuvent instituer une Église; ni l'un ni l'autre ne peuvent adopter de loi qui aiderait une religion, ou toutes les religions, ou qui préférerait l'une par rapport à l'autre », Everson v. Board of Education of the township of Ewing, 330 US1, 16 (1947).

54. Larson v. Valente, 456 US 228, 244 (1982), Church of the Lukumi Babalu Aye, Inc v. City of Hieleah, 508, Us 520, 525 (1993) sur la religion Santeria et ses sacrifices, Employment Division, Department of Human resources of Oregon v. Smith, 494 Us 872, 874 (1990).

55. Tout type de prière même aconfessionnelle (arrêt Engel v. Vitale de 1962, School District v. Schempp de 1963, Wallace v. Jaffrey de 1985, Lee v. Weissman de 1992). Pour une présentation exhaustive voir l'article de D. Laycok, «La religion et l'État aux États-Unis; affrontement des théories et changements historiques ", in La Conception américaine de la laïcité, la partie sur l'expression religieuse parrainée par le gouvernement, p. 56-62.

56. Arrêt Epperson v. Arkansas de 1968 et Edwards v. Aguillard de 1987.

57. Contry of Alleghency v. ACL, 492 US 573 (1989). Les grands arrêts de la Cour suprême des États-Unis ont été publiés en français par E. Zoller, in Grands arrêts de la Cour suprême des États-Unis, Paris, PUF, 2002.

58. Depuis la très célèbre interdiction de la bigamie mormone (Reynolds v. United States, 98 US 145, 167 (1879)), la tradition est établie du compelling state interest (intérêt d'État impérieux). Elle s'est appliquée aux vaccinations obligatoires, au dimanche jour de repos légal, à l'obligation du service militaire, à l'obligation de payer ses impôts, ses cotisations sociales, etc. Voir E. Zoller, «La laïcité aux États-Unis ou la séparation des Églises et de l'État dans la société pluraliste», p. 23.

59. Avec un célèbre arrêt, Sherbert v. Verner, 374 US 398 (1963).

60. 494 US 872 (1990).

61. Elle confirma à l'occasion la condamnation de l'archevêque de San Antonio qui avait outrepassé les règles sur la protection des monuments historiques pour agrandir une Église, arrêt City of Boern v. Flores, 521 US 507 (1997). Cette jurisprudence confirme que si les États peuvent accorder des exemptions au cas par cas à l'application de leurs lois, cette exemption n'est pas obligatoire et le principe reste qu'une loi générale s'applique aux religionnaires quand bien même elle contrarie l'exercice d'un culte. De plus, jamais une exemption ne peut être contraire à la Constitution et aux lois fédérales.

62. "Aucun impôt ne peut être levé pour soutenir des activités ou institutions religieuses ou pour enseigner ou pratiquer une religion, de quelque nom qu'on puisse les appeler et quelle qu'en puisse être la forme", arrêt Everson v. Board of Education of the Township of Ewing, 330 US 16 (1947).

63. Lemon. v. Kurtzman 403 US 602 (1971).

64. En l'occurrence le financement par le Rhode Island d'un supplément de salaire de $15 \%$ pour les professeurs de l'enseignement libre et le remboursement par la Pennsylvanie des frais d'équipement et de matériel engagés pour des activités non religieuses par les écoles confessionnelles.

65. Arrêt Mitchell v. Helms, 530 US 793 (2000) sur des équipements en ordinateur payés par la collectivité à la faveur d'un programme d'équipement scolaire, arrêt Mueller v. Allen, 463 US 388 (1983) sur les achats de livres et de fournitures scolaires.

66. Par le système du school voucher, au nom du true private chose, qui avantage indirectement les écoles privées. Ce système n'a pas été trouvé contraire au principe de séparation, arrêt Zelmann v. Simmons-Harris, 536 US 639, 649 (2002), ni non plus l'aide personnalisée aux jeunes aveugles scolarisés dans des écoles religieuses, arrêt Witters v. Washington Departement of Services for the Blind, 474 US 481 (1986).

67. Arrêt Agostini v. Felton, 521 US 203 (1997), qui admet la mise à disposition d'un enseignant du langage par signes pour un enfant sourd et muet d'une école privée.

68. Sur le financement des activités encadrées par des institutions religieuses, cf. E. Zoller, «La laïcité aux États-Unis ou la séparation des Églises et de l’État dans la société pluraliste», p. 19 et D. Laycock, «La religion et l'État aux États-Unis; affrontement des théories et changements historiques », p. 50-56: Laycock insiste sur le conflit politico-juridique autour de la neutralité de l'État, une partie des commentateurs souhaitant que l'État apporte un soutien financier non discriminatoire aux écoles religieuses et aux actions caritatives, l'autre partie défendant l'interdiction du financement public de toute 
toujours plus fort des convictions religieuses au détriment de la neutralité financière et éducative de l'éducation publique américaine.

De même certains projets de lois favorisent les groupements religieux, témoignage de la vigilance du religieux sur l'ordre politique. C'est ainsi que le réveil de la droite religieuse dans les années 1980 où se développent des lobbies fondamentalistes militant pour le retour légal de l'interdiction du divorce et de l'avortement, de même que le retour de l'enseignement créationniste et de la prière chrétienne à l'école, rappellent l'immixtion constante et facile du religieux dans le politique ${ }^{69}$ dans des débats qui sont loin d'être clos et agitent tous les jours la presse américaine.

Jusqu'à ce jour et sur ces questions, ces groupes n'ont pas obtenu gain de cause, ni sous la présidence de Reagan, ni sous celles des deux présidents Bush. Mais l’imprégnation de la sphère politique par des idéaux religieux aux États-Unis est comparable à l'imprégnation de la sphère politique par des idéaux agnostiques en France. Ils révèlent les tendances de la culture de chaque pays. Ils ne signifient pas que le droit leur est soumis. Ils ne signifient pas que l'État soit « confessionnel » d'un côté, ou "franc-maçon » de l'autre (même si c'est la caricature qui en ressort) et que les prises de position du président américain ou de son entourage en faveur de la prière à l'école seront suivies d'effets. Et de ce point de vue, il faut largement nuancer l'intrusion de la religion dans la sphère politique américaine, comme le fait Camille Froidevaux-Metterie dans ses recherches ${ }^{70}$, après l'échec du grand projet de politique sociale confiée aux associations caritatives confessionnelles, le Faith based and Communites Initiatives, par un transfert de fonds directs des services sociaux publics aux groupes confessionnels œuvrant dans ce sens. Le Community Solutions Act de l'été 2001, élaboré après un programme de réflexion très conséquent, s'est heurté à une très large coalition de "séparatistes » et de groupes religieux refusant que l'État s'immisce dans leurs affaires. Comme souvent - ce fut la même chose pour l'International Religious Freedom Act de $1998^{71}$ à l'origine suscité par les lobbies de droite religieuse auprès du Congrès puis tempéré dans son élaboration par un élargissement des intervenants depuis les rangs de lobbies plus modérés, libéraux et droits de l'homme - un compromis substantiel transforma ce projet en une loi prudente (CARE, Charity Aid, Recovery and Empowerment Act de 2002) qui rappela aux associations caritatives, qu'elles soient confessionnelles ou pas, leur obligation de respecter scrupuleusement le corpus législatif des droits civils ${ }^{72}$.

\section{Deux laïcités en concurrence?}

Il y a visiblement un rapport entre la crispation identitaire qui semble saisir la France depuis quelques années et son incapacité à matérialiser sa projection universaliste, incluant son modèle laïque ${ }^{73}$. Quelques nostalgiques peuvent se rappeler des révolutions françaises de 1830 et 1848 qui ont été passionnément suivies et imitées en leur temps par d'autres Européens avides de libertés et d'indépendance... Pendant que les Français se querellent et projettent leurs refus, la promotion et la défense des droits de l'homme sont désormais assurées à travers le monde par des $\mathrm{ONG}$ et des diplomates qui ne sont pas français. Au printemps 2005, l'opinion publique française a rejeté la Constitution européenne, y compris en ce que, pour certains, ce projet «n'était pas laïque». Les drapeaux républicains ont été mis en berne pour la mort de Jean-Paul II, déclenchant une polémique nationale au même moment où les médias français s'étonnaient que les révolutions de velours du Grand Est dussent tout au travail d'ONG et de clubs de réflexion d'outre-atlantique ${ }^{74}$. Quasiment rien dans ces grands mouvements de libération ne doit à l'aide ou à l'influence culturelle de la France, alors que $30 \%$ du budget du ministère français des Affaires étrangères sont consacrés à cette dimension ${ }^{75}$.

De leur côté, les États-Unis «sont partout». Partout parce qu'ils défendent leurs intérêts, que leurs moyens semblent immenses, mais également - fait nouveau parce qu'ils entendent exporter leur universalisme démocratique. Et dans cet universalisme, leur modèle laïque est implicitement mis en avant et exporté par une diplomatie très active, alors que les critiques internationales devant le

religion, contre toute prise de position de l'État. Voir également F.M. Gedicks, The Rhetoric of Church and State: A critical analysis of Religion Clause Jurisprudence, Durham, Duke University Press, 1995.

69. Sur cette question extraordinairement débattue aux États-Unis, voir la synthèse claire de M. Cristi, From Civil to Political Religion: The Intersection of Culture, Religion and Politics, Ontario, Wilfried Laurier University Press, 2001.

70. C. Froidevaux-Metterie, « Les États-Unis, une République laïque », p. 95

71. J. Gunn, "The United States and the Promotion of Freedom of Religion and Belief», chapitre XXXII du collectif Faciliting Freedom of Religion or Belief: A Desbook, W. Cole Durham, T. Lindhom et B. Thazib-Lie (dir.), Leyde, Martinus Nijhoff Publishers, 2004, p. 721-747; B. Chelini-Pont, «La diplomatie religieuse des États-Unis en Europe occidentale et sa réception française (1996-2002) ", in Quelle politique religieuse en Europe et en Méditerranée?..., p. 189-192 ; E. Pastor, «US International Religious Freedom Act of 1998: an European Perspective», in Eleventh Annual International Law and Religion Symposium (BYU-Provo, 3-5 octobre 2004), à paraître dans la Brigham Young University Law Review, vol. $2005,2$.

72. «Si la visée fondamentaliste consiste à réaffirmer le pouvoir de commandement de la religion à l'intérieur de sociétés qui fonctionnent en dehors d'elle, la tentative est vouée à l'échec. Comment en effet rendre au religieux sa fonction ancienne de définition des normes de l'espace social dans le cadre moderne de la séparation des sphères temporelle et spirituelle? Les impasses du programme Faith Based Initiatives impulsé par G. W. Bush constituent en ce sens l'une des illustrations les plus patentes du caractère vain, pour ne pas dire désespéré, de l'ambition fondamentaliste dans le contexte américain... Bush ne sera pas le fondateur de la première théocratie moderne» (C. Froidevaux-Metterie, «Les États-Unis, une République laïque », p. 97).

73. E. Decaux, «La diplomatie française des droits de l'homme», S. Sur, «La puissance et le rang revisité », et J.-J. Roche, «La France et l'Universel », ces trois articles in Archives françaises des relations internationales, 2000, www.france.diplomatie.fr/cap/1385.htlm.

74. L. Mandeville, «Les ONG américaines démocratisent l'ex-Urss », Le Figaro, 6 avril 2005.

75. D. Atkinson, «De l'exception culturelle à la diversité culturelle: les relations internationales au cœur d'une bataille planétaire », in Annuaire français des relations internationales, 2000, vol. I, www.france-diplomatie.fr/cap/1385.htlm. 
modèle laïque français sont aujourd'hui nombreuses. Le cœur de ces critiques vise l'engagement excessif de l'État français et une certaine définition de la citoyenneté qui limite abusivement l'expression religieuse des individus et exclut le moins ressemblant, au nom de sa propre compétence à gérer l'espace public et à lui conserver sa «neutralité ». En quelque sorte la neutralité de l'État se transforme en neutralisation de la société, aux antipodes justement du respect constitutionnel du pluralisme religieux ${ }^{76}$.

Ces critiques contre la tendance discriminante du système français qui permet facilement de susciter des lois qui empiètent, au nom de l'ordre public et de la laïcité éducative, sur le principe de séparation, ont été très vivement émises par des ONG de protection des droits de l'homme et d'autres ONG militant spécifiquement pour la liberté religieuse ${ }^{77}$. Au sein des instances internationales, le rapporteur spécial pour les Nations Unies s'est inquiété par exemple de la loi du 15 mars $2004^{78}$ de même que l'Assemblée parlementaire du Conseil de l'Europe ${ }^{79}$. Il est vrai que le cas Sahin contre Turquie, en cours de jugement définitif devant la Cour européenne des droits de l'homme, semble faire rebondir en faveur de la France la question de la légitimité de l'interdiction du voile dans un État démocratique, et conforter la position française en invoquant la laïcité de l'État turc ${ }^{80}$. Mais dans le même temps le Comité des droits de l'homme des Nations Unies a condamné la position de l'Ouzbekistan ${ }^{81}$ sur cette épineuse question.

Nous ne pouvons sous-estimer l'importance du modèle de laïcité dans l'image de la France et des ÉtatsUnis, parce que le modèle démocratique de demain porte essentiellement sur l'articulation idoine du religieux dans la vie des individus et dans la sphère publique. La question de la liberté des personnes à « choisir » et à manifester leur religion y est centrale. Les critiques extérieures sur la laïcité française frappent souvent juste - quand elles portent sur les rigidités que véhicule «l'idéal laïque » dans un monde où les sociétés civiles se détachent de l'État comme instrument de leur cohésion et deviennent des acteurs à part entière du droit et de l'action politique. Elles mettent surtout en évidence le déclin d'un modèle qui n'est plus suffisamment porté ou crédible, quand de leur côté les juristes et diplomates américains investissent temps, argent, réflexions et rencontres autour de l'axe Religion et Droits de l'homme ${ }^{82}$. Certes, le modèle laïque français recule aussi parce que l'intégration européenne relativise son caractère exceptionnel ${ }^{83}$ et dégage, sous les effets de la jurisprudence de la Cour européenne, une laïcité « culturelle » à l'européenne - selon l'analyse proposée par Jean-Paul Willaimemoins séparatiste ${ }^{84}$. Mais la vraie raison - nous semblet-il-d'un désintérêt international pour le modèle français résulte de ce que le modèle américain s’impose comme le bon modèle à prétention universaliste, plus efficace dans sa diffusion et plus simple dans ses motivations : ce modèle accommodant aux religions des individus, intraitable sur leur caractère "privé», très sensible au droit de changer de religion (et pour cause, la religion est une affaire de volonté individuelle), devient clairement - s'il ne l'est déjà - l'étalon ou le socle principiel de la bonne démocratie, comme le souligne l'importance de cette vision dans les instances internationales comme l'OSCE, doté d'un Advisory Panel on Religious Freedom aux travaux nombreux et aux interventions constantes in situ.

Les États-Unis donnent à voir au monde leur exemplarité dans la promotion et la défense des droits de l'homme. Est-ce seulement l'effet de leur puissance et d'une imposition impérialiste? Leur puissance, à l'instar de la France à la fin du XVII ${ }^{\mathrm{e}}$ siècle, produit-elle un effet de rayonnement? Si nous tentons d'élaborer une hypothèse, nous dirions que

76. Dans le collectif The French Exception, E. Godin et T. Chafer (éd.), New York - Oxford, Berghahn Books, 2004, la définition analytique de cette exception place également en premier le dirigisme républicain de l'État qui domine souverainement la société civile, et joue un rôle de régulateur impératif qui refuse, au nom de l'individualité citoyenne, de reconnaître d'autres identités collectives, comme les minorités religieuses, linguistiques, culturelles ou régionales: "The relunctance of President Chirac to sign the European Charter for Regional or Minority Languages and the next legislation to ban the wearing of the veils in schools by muslims girls testify the continuing durability of this model ", p. XV de l'introduction, faisant allusion à l'ouvrage de P. Rosanvallon, Le Modèle politique français, Paris, Seuil, 2004. Pour une compréhension positive du rôle de l'État comme "instrument du laos », voir G. Haarscher, «La neutralité, l'intégrité et la protection des minorités », in L'État au Xx siècle, S. Goyard-Fabre (dir.), Paris, Vrin, 2004, p. $249-272$.

77. Par exemple et parmi un éventail important de rapports et commentaires, le communiqué de la Fédération internationale d’Helsinki pour les droits de l'homme du 17 décembre 2003, www. ihf-hr. org.

78. Rapport de l'ONU (services du rapporteur spécial) sur la liberté de religion dans le monde, du 27 octobre 2004, dans lequel la France figure sur une liste de 28 États où la liberté de conviction est minorée. Sur la doctrine onusienne en matière de liberté religieuse voir le rapport intermédiaire sur l'élimination de toutes les formes d'intolérance et de discrimination fondées sur la religion et la conviction, établi par le rapporteur Abdelfattah Amor, conformément à la résolution 54 / 159 de l'Assemblée générale, en date du 17 décembre 1999, portant sur la visite effectuée en Turquie du 30 novembre au 9 décembre 200o, additif 1 de la cinquantième session de l'Assemblée générale, 11 août 2000, A / 55 / 280.add.1. Voir enfin C. de Jong, The Freedom of Thougt, Conscience and Religion or Belief in the United Nations (1946-1992), Anvers, School of Human Rights Research Series - Intersentia-Hart, 200o.

79. Résolution 1309 (2002).

80. Laïcité que le premier jugement de la Cour ne définit pas, si ce n’est en référence à la laïcité française, quand bien même une étude attentive du système turc ne permet pas de conclure à la neutralité et à la « séparation» de l'État turc selon les critères franco-américains. Voir sur le site www. strasbourgconference.org, l'intervention du Professeur J. Gunn sur le cas Sahin, «Fearful Symbol, Islamic Headscarf and the European Court of Human Rights », voir également le texte de T. Lindholm, «The Strasbourg Court dealing with Turkey and the Human Right to Freedom of Religion or Belief».

81. Constatation du Comité des droits de l'homme des Nations Unies, 5 novembre 2004, Communication n ${ }^{\circ} 931 / 2000$ consultable sur internet.

82. Une étude exhaustive de l'activité universitaire internationale sur cette question, souvent financée ou cooptée par des associations ou des ONG militantes reste à faire. Pour une réflexion théorique sur cette question, voir J. Gunn, « The Complexity of Religion and the Definition of Religion in International Law ", Harvard Human Rights Journal, vol. 16, printemps 2003, p. 191-216.

83. O. Dord, Laïcité: le modèle français sous influence européenne, Paris, Fondation Robert Schuman, 2004.

84. «Une France qui, forte de sa laïcité, percevrait les autres pays d'Europe comme quelque peu attardés parce qu'insuffisamment émancipés par rapport à la religion serait très mal perçue par nos partenaires qui, dans ce domaine comme dans d'autres, estiment ne pas avoir de leçons à recevoir » (Introduction du livre de J.-P. Willaime, Europe et Religions, les enjeux du xxi siècle, Paris, Fayard, 2004). 
les États-Unis se considèrent à la pointe actuelle du respect des droits de l'homme dans leur compréhension des droits de l'homme spirituel ${ }^{85}$. De la même manière que la France proposait un modèle d'humanisme kantien exemplaire face à l'athéisme des grandes idéologies ou à la structure théocratique et autoritaire des sociétés traditionnelles, les États-Unis d'aujourd'hui projettent un humanisme " sacré », prêt à répondre aux débordements antidémocratiques des radicalismes religieux, comme à l'activisme antireligieux et persécuteur des États non démocratiques.

Le système international, avec la fin de la Guerre froide, a changé de paradigme métaphysique: dans le sillage de la domination européenne et de la modernité politique qu'elle portait, il a semblé dans la première moitié du $\mathrm{xx}^{\mathrm{e}}$ siècle que la religion n'avait plus d'avenir. L'ère des idéologies qui en a découlé a fait se confondre la projection d'un modèle humaniste à l'athéisme soft avec la neutralité de l'État et de l'espace public au service de tous les citoyens. Après le temps du désenchantement du monde, nous voici passés dans le temps de son réenchantement ${ }^{86}$ globalisé. Au temps du "désenchantement», la France servait de lumière positive, jusque et même dans ses colonies en révolte. Elle inspirait l'Esprit des Nations souveraines et des Peuples libres. La laïcité française représentait le contre- feu humaniste qui ne basculait pas devant les terribles dérives des totalitarismes persécuteurs et des avatars insurrectionnels de la Guerre froide. Mais elle représentait l'âme philosophique d'un monde positif vidant le ciel de ses mystères. La foi a supplanté la raison, comme paradigme opérant, rassembleur des nouveaux humanistes, dans cette ère de l'hyperpuissance américaine, où les grandes visions laïques et messianiques que furent le nationalisme, le socialisme et le communisme, ont été balayées par des idéologies religieuses intolérantes et systématiques, comme l'islamisme, les fondamentalismes juif et chrétiens ou l'hindouïsme politique. Les États-Unis sont en train de se transformer activement et consciemment en lumière d'un temps où la religion n'est plus une question privée sans répercussion politique, mais une question centrale de l'avancée démocratique. Ils proposent au monde leur propre expérience et une vision d'une Nation respectueuse de tous les droits humains, y compris les spirituels, où les citoyens pratiquent une vigilance de tous les instants pour les conserver et les améliorer, atteignant le cœur des interrogations actuelles sur la conciliation de la démocratie et de la religion ${ }^{87}$. Resteront-ils les seuls à faire une telle proposition?

85. R. Letteron, «L'universalité des droits de l'homme: apparences et réalités. L'idéologie des droits de l'homme en France et aux États-Unis », Annuaire français des relations internationales, 2001, www.France-diplomatie.fr/cap/1385.htlm; D. Decherf, «Les États-Unis au secours des droits de l'homme religieux ", Critique internationale, $\mathrm{n}^{\circ}$ 15, avril 2002.

86. P. Berger, Le Réenchantement du monde, Paris, Bayard, 2002.

87. G. Parmentier, « George W. Bush : le croisé de la démocratie », Politique internationale, nº 107, printemps 2005, p. 147-160. 